\title{
Identification of Molecular Biomarkers in Taiwanese Patients with Wilms Tumor Using a Methylation-Specific Multiplex Ligation-Dependent Probe Amplification (MS-MLPA)-Based Approach
}

\author{
Meng-Yao Lu \\ National Taiwan University Hospital
}

Wen-Chung Wang

Jen-Ai Hospital Dali Branch

Tai-Cheng Hou

Jen-Ai Hospital Dali Branch

Chen-Yun Kuo

Jen-Ai Hospital Dali Branch

Yen-Chein Lai ( $\nabla$ yenchein@csmu.edu.tw)

Chung Shan Medical University https://orcid.org/0000-0001-9072-5272

\section{Research}

Keywords: Methylation detection, Molecular pathology, Multiplex ligation-dependent probe amplification, Nephroblastoma, Tumor suppressor gene, Wilms tumor

Posted Date: September 28th, 2020

DOI: https://doi.org/10.21203/rs.3.rs-80694/v1

License: (c) (i) This work is licensed under a Creative Commons Attribution 4.0 International License. Read Full License 


\section{Abstract}

Background: Wilms tumor is a solid tumor that frequently occurs in children. Genetic or epigenetic aberrations in WT1 and WT2 loci are implicated in its etiology. Moreover, tumor suppressor genes are frequently silenced by methylation in this tumor.

Methods: In the present study, we analyzed the methylation statuses of promoter regions of 24 different tumor suppressor genes using a methylation-specific multiplex ligation-dependent probe amplification (MS-MLPA)-based approach in six Wilms tumors.

Results: All six Wilms tumors showed methylation of RASSF1 specific to tumors, not in normal tissues. Moreover, methylated HIC1 was identified in stromal type Wilms tumors and methylated BRCA1 was identified in epithelial type Wilms tumors. Unmethylated CASP8, RARB, MLH1_167, APC, and CDKN2A were found only in blastemal predominant type Wilms tumors.

Conclusions: Our results indicated that methylation of RASSF 1 is the essential event in the tumorigenesis of Wilms tumor, which may inform its clinical and therapeutic management. In addition, mixed type Wilms tumors may be classified according to epithelial, stromal, and blastemal components via MS-MLPA-based approach.

\section{Background}

Wilms tumor, also known as nephroblastoma, is an embryonal malignant tumor of the kidney. It is the most commonly occurring solid tumor in children, excluding brain tumors [1-3]. The etiology of Wilms tumor is still poorly understood, although a number of associated genes and loci have been identified $[4,5]$. The genetic changes in Wilms tumors are diverse and involve approximately 40 genes [6]. Genes that have been previously implicated include WT1, CTNNB1, FAM123B, DROSHA, DGCR8, XPO5, DICER1, SIX1, SIX2, MLLT1, MYCN, and TP53. Whole-genome sequencing and wholeexome sequencing have led to the addition of BCOR, BCORL 1, NONO, MAX, COL6A3, ASXL 1, MAP3K4, and ARID1A [7]. Recently, four new predisposing genes have been identified - TRIM28, FBXW7, NYNRIN, and KDM3B [5].

Hypermethylation of $\mathrm{CpG}$ islands upstream of tumor suppressor genes has been reported for which methylation status is altered in one or more types of tumors $[8,9]$. It is interesting to note that Wilms tumor develops mainly through alterations in epigenetic regulation triggered by dedifferentiation [10]. However, the risk of Wilms tumor conferred by epigenetic changes associated with tumor suppressor genes is poorly characterized. In the present study, we investigated the methylation statuses of promoter regions of 24 different tumor suppressor genes in six Wilms tumors using a methylationspecific multiplex ligation-dependent probe amplification (MS-MLPA)-based approach.

\section{Methods}

\section{Study subjects}

The six subjects have been described elsewhere [11-13]. Their paraffin-embedded Wilms tumor tissue samples were provided by the Department of Pediatrics of National Taiwan University Hospital. These patients had no history of DenysDrash syndrome, Frasier syndrome, or Beckwith-Wiedemann syndrome. The study procedures were approved by the Institutional Review Board of Chung Shan Medical University Hospital (reference number CS2-16003). All procedures that involved human participants were conducted in accordance with the ethical standards of the institutional and/or national research committee and the 1964 Declaration of Helsinki and its later amendments or comparable ethical standards.

\section{Histological examination}


The tumor tissue samples (W7 to W12) were embedded in paraffin wax and cut into 5 um-thick slices. Then, sections were stained with hematoxylin and eosin and reviewed by two of the authors (T-C Hou and C-Y Kuo) under a microscope to confirm their diagnostic classifications. Typing of Wilms tumor has been described in detail elsewhere [11, 13]. W7 is of blastemal type and W8 is of epithelial type. In addition, W10 and W11 are of stromal type. W9 is a triphasic Wilms tumor comprised of three components: blastema, stroma, and epithelium, while W12 is of mixed blastemal and epithelial type.

\section{DNA extraction}

Genomic DNA was purified from paraffin-embedded tissues with the DNA FFPE Tissue Kit (Qiagen), according to the manufacturer's instructions and dissolved in $100 \mu \mathrm{l}$ of TE buffer (10 mM Tris- $\mathrm{HCl}, \mathrm{pH}$ 8.0, and $1 \mathrm{mM}$ EDTA) as previously described $[11,13]$. UV-Vis measurements of DNA concentration of each sample were obtained using NanoDrop UV-VIS Spectrophotometer (Thermo Scientific Nano-Drop 2000c).

\section{Methylation analysis}

MS-MLPA analysis was performed using Salsa MS-MLPA kit ME001-C2 Tumor suppressor-1 (MRC-Holland) according to the manufacturer's instructions. Samples were then subjected to capillary electrophoresis on an ABI PRISM 3130XL (Applied Biosystems). Twenty-six MS-MLPA probes were used to detect the methylation statuses of promoter regions of 24 different tumor suppressor genes by Hhal digestion (Table 1). MLPA results were analyzed using GeneMarker version 3.2.1 (SoftGenetics, LLC) to determine copy numbers and methylation statuses of the Hhal sites. The internal methylation ratio was obtained by comparison of the Hhal digested aliquot (Fig. 1B) with the paired undigested aliquot (Fig. 1A) from each sample with intra-sample data normalization according to the manufacturer's instructions [14]. Methylation was assessed by comparing the probe methylation percentages of the test sample with the percentages of the 5 normal reference samples. Copy number ratio of 1.0 and methylation ratio of 0 were expected in most genes on normal reference, meaning that the methylation compared to normal reference was unlimited $(\infty)$. If methylation ratios of test sample and normal reference samples were appropriate, methylation compared to normal reference was around 1.0. 
Table 1

Chromosomal locations of the 41 probes in ME001-C2 Tumor suppressor-1.

\begin{tabular}{|c|c|c|c|c|c|}
\hline Length (NT) & Gene & MLPA probe & Hhal site & Chromosomal location & $\begin{array}{l}\text { MV location } \\
\text { (hg18) }\end{array}$ \\
\hline 400 & TP73 & 04050-L01263 & yes & 1p36.32 & 01-003.558977 \\
\hline 265 & CASP8 & 02761-L02210 & yes & $2 q 33.1$ & 02-201.830871 \\
\hline 353 & VHL & 03810-L01211 & yes & $3 p 25.3$ & $03-010.158426$ \\
\hline 193 & RARB & 04040-L01698 & yes & $3 p 24.2$ & $03-025.444559$ \\
\hline 167 & MLH1 & 01686-L01266 & yes & 3p22.2 & 03-037.009621 \\
\hline 463 & MLH1 & 02260-L01747 & yes & $3 p 22.2$ & 03-037.010000 \\
\hline 475 & CTNNB1 & 03984-L03251 & - & $3 p 22.1$ & $03-041.241066$ \\
\hline 328 & RASSF1 & 02248-L01734 & yes & $3 p 21.31$ & $03-050.353298$ \\
\hline 382 & RASSF1 & 03807-L02159 & yes & $3 p 21.31$ & $03-050.353347$ \\
\hline 409 & FHIT & 02201-L01699 & yes & $3 p 14.2$ & 03-061.211918 \\
\hline 483 & CASR & 02683-L02148 & - & $3 q 21.1$ & $03-123.485226$ \\
\hline 148 & APC & 01905-L01968 & yes & $5 q 22.2$ & 05-112.101357 \\
\hline 373 & ESR1 & 02202-L01700 & yes & $6 q 25.1$ & 06-152.170883 \\
\hline 154 & PARK2 & 03366-L02750 & - & $6 q 26$ & $06-162.126766$ \\
\hline 310 & CDK6 & 03184-L02523 & - & 7q21.3 & 07-092.085391 \\
\hline 161 & CDKN2A & 01524-L01744 & yes & $9 p 21.3$ & 09-021.985276 \\
\hline 211 & CDKN2B & 00607-L00591 & yes & $9 p 21.3$ & 09-021.998808 \\
\hline 346 & DAPK1 & 01677-L01257 & - & $9 q 21.33$ & 09-089.303075 \\
\hline 364 & LOC254312 & 01234-L00781 & yes & 10p14 & $10-011.017023$ \\
\hline 136 & CREM & 00981-L00566 & - & 10p12.1 & $10-035.517225$ \\
\hline 292 & KLLN & 02203-L08261 & yes & $10 q 23.3$ & $10-089.612348$ \\
\hline 319 & CD44 & 03817-L01731 & yes & $11 \mathrm{p} 13$ & $11-035.117389$ \\
\hline 454 & GSTP1 & 01638-L01176 & yes & $11 q 13.2$ & $11-067.107774$ \\
\hline 184 & ATM & 04044-L03849 & yes & $11 q 22.3$ & $11-107.599044$ \\
\hline 427 & CADM1 & 03819-L03848 & yes & $11 q 23.3$ & $11-114.880585$ \\
\hline 175 & TNFRSF1A & 00554-L13516 & - & $12 \mathrm{p} 13$ & $12-006.321241$ \\
\hline 444 & CD27 & 00678-L00124 & - & 12p13.31 & $12-006.430685$ \\
\hline 274 & CDKN1B & 07949-L07730 & yes & $12 \mathrm{p} 13.1$ & $12-012.761863$ \\
\hline 229 & PAH & 02334-L01820 & - & $12 \mathrm{q} 23.2$ & $12-101.795401$ \\
\hline 238 & CHFR & 03813-L03753 & yes & $12 q 24.33$ & $12-131.974372$ \\
\hline
\end{tabular}




\begin{tabular}{|llllll|}
\hline Length (NT) & Gene & MLPA probe & Hhal site & Chromosomal location & $\begin{array}{l}\text { MV location } \\
\text { (hg18) }\end{array}$ \\
\hline 301 & & & & & $13-031.787722$ \\
\hline 418 & BRCA2 & $04042-L 03755$ & yes & $13 q 12.3$ & $13-031.851548$ \\
\hline 202 & BRCA2 & $01617-L 01199$ & - & $13 q 13.1$ & $14-074.578836$ \\
\hline 281 & MLH3 & $01245-L 00793$ & - & $14 q 24.3$ & $16-002.061786$ \\
\hline 337 & TSC2 & $01832-L 01397$ & - & $16 p 13.3$ & $16-067.424755$ \\
\hline 436 & CDH1 & $02416-L 01862$ & - & $16 q 22.1$ & $16-081.218219$ \\
\hline 220 & CDH13 & $07946-L 07727$ & yes & $16 q 23.3$ & $17-001.905107$ \\
\hline 246 & HIC1 & $03804-L 00949$ & yes & $17 p 13.3$ & $17-038.530811$ \\
\hline 256 & BRCA1 & $05162-L 04543$ & yes & $17 q 21.31$ & $18-058.946868$ \\
\hline 390 & BCL2 & $00587-L 00382$ & - & $18 q 21.33$ & $19-056.050014$ \\
\hline 142 & KLK3 & $00713-L 00108$ & - & $19 q 13.33$ & $22-031.527795$ \\
\hline
\end{tabular}

\section{Results}

The methylation statuses of the Hhal sites in 24 different tumor suppressor genes were determined by 26 MS-MLPA probes on MS-MLPA analysis (Fig. 1). If the target site was unmethylated, the DNA-probe complex was digested to prevent exponential amplification. No signal was detected after fragment analysis (Fig. 1B).

Methylation of RASSF1 and CDKN2B was found in all six Wilms tumors (Fig. 1A, Table 2). Methylation was observed in both probes in RASSF1 gene. Internal methylation ratio was not 0 and methylation compared to normal reference was unlimited $(\infty)$. CDKN2B gene was methylated in normal reference. In comparison with normal reference, methylation was not unlimited $(\infty)$. W7 to W11 methylation was 3.72, 8.85, 2.58, 3.15, and 5.40-fold that of normal reference, respectively. However, W12 methylation was only 1.05 -fold that of normal reference. 
Table 2

Methylated and unmethylated genes identified in six Wilms tumors

\begin{tabular}{|c|c|c|c|c|c|c|c|c|}
\hline Size (NT) & Gene & Cytoband & W7 & W8 & W9 & W10 & W11 & W12 \\
\hline \multicolumn{9}{|l|}{ All } \\
\hline 328 & RASSF1 & $3 p 21.31$ & $0.84 / \infty$ & $0.17 / \infty$ & $0.74 / \infty$ & $0.74 / \infty$ & $0.28 / \infty$ & $0.63 / \infty$ \\
\hline 382 & RASSF1 & $3 p 21.31$ & $0.94 / \infty$ & $0.22 / \infty$ & $0.74 / \infty$ & $0.71 / \infty$ & $0.33 / \infty$ & $0.70 / \infty$ \\
\hline 211 & $C D K N 2 B$ & $9 p 21.3$ & $0.18 / 3.72$ & $0.54 / 8.85$ & $0.15 / 2.58$ & $0.13 / 3.15$ & $0.26 / 5.40$ & $0.073 / 1.05$ \\
\hline \multicolumn{9}{|l|}{ None } \\
\hline 353 & $V H L$ & 3p25.3 & $0 / 0$ & $0 / 0$ & $0 / 0$ & $0 / 0$ & $0 / 0$ & $0 / 0$ \\
\hline 463 & $M L H 1$ & $3 p 22.2$ & $0 / 0$ & $0 / 0$ & $0 / 0$ & $0 / 0$ & $0 / 0$ & $0 / 0$ \\
\hline 409 & FHIT & 3p14.2 & $0 / 0$ & $0 / 0$ & $0 / 0$ & $0 / 0$ & $0 / 0$ & $0 / 0$ \\
\hline 238 & CHFR & $12 q 24.33$ & $0 / 0$ & $0 / 0$ & $0 / 0$ & $0 / 0$ & $0 / 0$ & $0 / 0$ \\
\hline 301 & $B R C A 2$ & $13 q 12.3$ & $0 / 0$ & $0 / 0$ & $0 / 0$ & $0 / 0$ & $0 / 0$ & $0 / 0$ \\
\hline \multicolumn{9}{|l|}{$\begin{array}{l}\text { Stroma } \\
\text { only }\end{array}$} \\
\hline 220 & HIC1 & 17p13.3 & $0 / 0$ & $0 / 0$ & $0.05 / \infty$ & $0.06 / \infty$ & $0.04 / \infty$ & $0 / 0$ \\
\hline \multicolumn{9}{|l|}{$\begin{array}{l}\text { Epithelium } \\
\text { only }\end{array}$} \\
\hline 246 & $B R C A 1$ & $17 q 21.31$ & $0 / 0$ & $0.04 / \infty$ & $0.03 / \infty$ & $0 / 0$ & $0 / 0$ & $0.03 / \infty$ \\
\hline \multicolumn{9}{|l|}{$\begin{array}{l}\text { Blastema } \\
\text { only }\end{array}$} \\
\hline 265 & CASP8 & $2 q 33.1$ & $0 / 0$ & $1.01 / \infty$ & $0.79 / \infty$ & $0.58 / \infty$ & $0.55 / \infty$ & $0.79 / \infty$ \\
\hline 193 & $R A R B$ & $3 p 24.2$ & $0 / 0$ & $0.05 / 9.28$ & $0.10 / 11.75$ & $0.12 / 23.95$ & $0.06 / 8.18$ & $0.09 / 10.25$ \\
\hline 167 & $M L H 1$ & 3p22.2 & $0 / 0$ & $0.03 / 2.82$ & $0.09 / 4.62$ & $0.06 / 3.863$ & $0.03 / 2.79$ & $0.05 / 2.96$ \\
\hline 148 & $A P C$ & $5 q 22.2$ & $0 / 0$ & $0.06 / \infty$ & $0.15 / \infty$ & $0.09 / \infty$ & $0.08 / \infty$ & $0.15 / \infty$ \\
\hline 161 & $C D K N 2 A$ & $9 p 21.3$ & $0 / 0$ & $0.05 / \infty$ & $0.06 / \infty$ & $0.09 / \infty$ & $0.04 / \infty$ & $0.07 / \infty$ \\
\hline \multicolumn{9}{|l|}{ Others } \\
\hline 184 & ATM & $11 q 22.3$ & $0 / 0$ & $0.04 / \infty$ & $0.10 / \infty$ & $0.10 / \infty$ & $0.05 / \infty$ & $0.18 / \infty$ \\
\hline 400 & TP73 & 1p36.32 & $0 / 0$ & $0.09 / \infty$ & $0.12 / \infty$ & $0.13 / \infty$ & $0 / 0$ & $0.10 / \infty$ \\
\hline 454 & GSTP1 & 11q13.2 & $0 / 0$ & $0.07 / \infty$ & $0.09 / \infty$ & $0.09 / \infty$ & $0 / 0$ & $0.09 / \infty$ \\
\hline 292 & $K L L N$ & $10 q 23.3$ & $0.13 / \infty$ & $0.07 / \infty$ & $0.07 / \infty$ & $0 / 0$ & $0.05 / \infty$ & $0 / 0$ \\
\hline 436 & $\mathrm{CDH} 13$ & $16 q 23.3$ & $0 / 0$ & $0.08 / \infty$ & $0.09 / \infty$ & $0.11 / \infty$ & $0 / 0$ & $0.10 / \infty$ \\
\hline 373 & ESR1 & $6 q 25.1$ & $0 / 0$ & $0.08 / \infty$ & $0 / 0$ & $0.13 / \infty$ & $0 / 0$ & $0 / 0$ \\
\hline 319 & $C D 44$ & $11 \mathrm{p} 13$ & $0.34 / \infty$ & $0 / 0$ & $0 / 0$ & $0.07 / \infty$ & $0 / 0$ & $0 / 0$ \\
\hline 142 & TIMP3 & $22 q 12.3$ & $0 / 0$ & $0.04 / \infty$ & $0.05 / \infty$ & $0 / 0$ & $0 / 0$ & $0 / 0$ \\
\hline
\end{tabular}

Data are presented as internal methylation ratio/methylation compared to normal reference. 


\begin{tabular}{|lllllllll|}
\hline Size (NT) & Gene & Cytoband & W7 & W8 & W9 & W10 & W11 & W12 \\
\hline 346 & DAPK1 & $9 \mathrm{q} 21.33$ & $0 / 0$ & $0.041 / \infty$ & $0 / 0$ & $0 / 0$ & $0 / 0$ & $0 / 0$ \\
\hline 427 & CADM1 & $11 \mathrm{q} 23.3$ & $0 / 0$ & $0.06 / \infty$ & $0 / 0$ & $0 / 0$ & $0 / 0$ & $0 / 0$ \\
\hline 274 & CDKN1B & $12 \mathrm{p} 13.1$ & $0 / 0$ & $0.04 / \infty$ & $0 / 0$ & $0 / 0$ & $0 / 0$ & $0 / 0$ \\
\hline
\end{tabular}

VHL, MLH1_463, FHIT1, CHFR, and BRCA2 were unmethylated in all six Wilms tumors (Fig. 1B). Moreover, there was methylation of HIC1 in the Wilms tumors of stromal type (W9, W10, and W11) and methylation of BRCA1 in the Wilms tumors of epithelial type (W8, W9, and W12). CASP8, RARB, MLH1_167, APC, and CDKN2A were unmethylated only in W7, which was of blastemal predominant type (Table 2). In other words, there was methylation of CASP8, RARB, MLH1_167, $A P C$, and $C D K N 2 A$ in stromal and epithelial type Wilms tumors, but not in blastemal type Wilms tumors.

In addition to these 13 tumor suppressor genes (15 MS-MLPA probes), there were differential methylation patterns for the other 11 tumor suppressor genes among the six Wilms tumors. The six Wilms tumors are listed in order from minimum number to maximum number of these methylated genes: W7 and W11 (2), W12 (4), W9 and W10 (6), and W8 (10). Only two tumor suppressor genes, $K L L N$ and $C D 44$, were methylated in W7. Only one tumor suppressor gene, $C D 44$, was unmethylated in W8.

\section{Discussion}

Traditional methods of methylation detection involve modifying DNA by methylation-specific restriction enzymes or sodium sulfite, combined with Sanger sequencing, PCR, or hybridization analysis. This process is cumbersome and difficult, with low reproducibility and insufficient sensitivity. MLPA is a PCR amplification reaction. ME001 can simultaneously detect changes in the degree of methylation of up to 24 different genes in a single reaction tube. Due to MLPA's easy operation, low cost, and wide range of applications, our team has published several studies based on this method $[13,15,16]$. However, MLPA analysis of tumor samples only provides information regarding the "average" situation in the cells from which the DNA samples were purified [14].

The results of this study regarding methylation of RASSF1 gene are consistent with the findings of previous studies [17, 18]. RASSF1 gene on 3p21.31 encodes a cytosolic RASSF1A protein similar to Ras effector proteins [19]. De novo methylation of the RASSF1 promoter is one of the most frequent epigenetic inactivation events in human cancer and leads to silencing of RASSF1 expression [20,21]. Association of RASSF1 promoter methylation with Wilms tumor has been reported [22]. The methylation status of RASSF1 might be a novel biomarker for predicting outcome of Wilms tumor patients [23]. It appears that methylation of RASSF1 is the essential event in the tumorigenesis of Wilms tumor, which may inform its diagnostic, clinical, and therapeutic management. CDKN2B gene hypermethylation was observed in W7 to W11, but not in W12. CDKN2B gene on 9p21.3 encodes the p1 $5^{\mathrm{INK} 4 \mathrm{~B}}$ protein that binds to CDK4 or CDK6 and inhibits its activation [24]. Hypermethylation of $C D K N 2 B C p G$ islands occurs in the majority of leukemia patients [25].

Hypermethylation at $\mathrm{CpG}$ islands in the 5 ' ends of tumor suppressor genes is controversial and difficult to interpret. HIC1 gene on 17p13.3 encodes a transcriptional repressor for p21 [26]. Hypermethylation of HIC1 gene is found in 3\% of Wilms tumors [27]. CASP8 gene on 2q33.1 encodes Caspase-8 that is an apoptosis-related cysteine peptidase [28]. In 43\% of Wilms tumors there is methylation at CASP8 [22]. MLH1 gene on 3p22.2 encodes proteins that detect and repair DNA mismatches [29]. A small proportion of Wilms tumors might be associated with the presence of microsatellite instability [30]. CDKN2A gene on 9p21.3 encodes two proteins that regulate two critical cell cycle regulatory pathways, the p53 pathway and the RB1 pathway [31]. Arcellana-Panlilio et al. demonstrated methylation of the CpG island in the 5' region of $C D K N 2 A$ (p16) in seven out of seven Wilms tumors exhibiting decreased $C D K N 2 A$ expression [32]. This is inconsistent with 
the finding of negligible methylation of the 5' $\mathrm{CpG}$ island of $C D K N 2 A$ by Erlich et al. [21]. BRCA1 gene on 17q21.31 encodes a nuclear phosphoprotein that plays a role in maintaining genomic stability [33]. However, $B R C A 1$, which demonstrates promoter hypomethylation, is over-expressed in Wilms tumor [34]. RARB2 gene on 3p24.2 encodes retinoic acid receptor beta that is a type of nuclear receptor activated by all-trans retinoic acid and 9-cis retinoic acid [35]. Our results were inconsistent with those of Morris et al. in which promoter methylation was absent at RARB2 [22]. APC gene on 5q22.2 encodes a 312-kDa protein that acts as an antagonist of the Wnt signaling pathway [36]. Activation of the Wnt/ $\beta$-catenin pathway is common in Wilms tumor, but rarely through $\beta$-catenin mutation and $A P C$ promoter methylation [37]. An important finding of this study is the possibility to further classify mixed type Wilms tumors using genetic results of epithelial, stromal, and blastemal components based on MS-MLPA-based approach. In particular, the methylation statuses of these 9 genes make them candidate molecular markers of metastasis in Wilms tumors.

In Wilms tumor, the differentiation arrest of renal progenitor cells is not complete, allowing for maturing lineages of varying proportions [7]. The outcome for stromal and epithelial predominant Wilms tumors is generally excellent [38]. Histological classification of Wilms tumor is not always possible based on morphology alone [39]. From our results, methylation of HIC1 in stroma, BRCA1 in epithelium, and CASP8, RARB, MLH1_167, APC, and CDKN2A in either stroma or epithelium can be used to identify stromal predominant and epithelial predominant Wilms tumors which are associated with a good outcome [38]. In the situation that the subtype of Wilms tumor is difficult to diagnose, unmethylated CASP8, RARB, MLH1_167, APC, and CDKN2A are potential diagnostic markers to diagnose blastemal type Wilms tumors which are associated with a poor outcome [40]. Stratification of Wilms tumor by epigenetic analysis of these genes highlights the benefits of methylation status analysis of important tumor suppressor genes. Is it possible that epigenetic modifications in Wilms tumor provide potential therapeutic options? To answer this question, additional studies based on a larger number of cases and tissue microdissection are necessary.

\section{Conclusions}

In summary, all six Wilms tumors showed methylation of RASSF1 specific to tumors, not in normal tissues. Moreover, methylation of HIC1 was identified in the Wilms tumors of stromal type and methylation of BRCA1 was identified in the Wilms tumors of epithelial type. Unmethylated CASP8, RARB, MLH1_167, APC, and CDKN2A was found only in the Wilms tumor that was of blastemal predominant type. Our results indicated that methylation of RASSF1 is the essential event in the tumorigenesis of Wilms tumor, which may inform its clinical and therapeutic management. In addition, mixed type Wilms tumors may be classified using genetic results of epithelial, stromal, and blastemal components based on MSMLPA-based approach. However, a larger number of cases are necessary to further refine the molecular classification and pathogenesis of Wilms tumors.

\section{Abbreviations}

$A P C$. adenomatous polyposis coli; $B R C A 1$ : breast cancer 1; BRCA2: breast cancer 2; CASP8: caspase 8; CDKN2A: cyclin dependent kinase inhibitor 2A; CDKN2B: cyclin dependent kinase inhibitor 2B; CHFR: Checkpoint with Forkhead and Ring Finger Domains; DNA: deoxyribonucleic acid; FHIT: fragile histidine triad protein; HIC1: hypermethylated-in-cancer 1; KLLN: killin; MLH1: mutL homolog 1; MS-MLPA: methylation-specific multiplex ligation-dependent probe amplification; NT: nucleotide; RARB2: retinoic acid receptor B2; RASSF1: ras-association domain family member 1; UV-VIS: ultraviolet-visible; $V H L$ : von Hippel-Lindau.

\section{Declarations}

\section{Acknowledgments}


The authors would like to thank GenePhile Bioscience Laboratory of Ko's Obstetrics and Gynecology Clinic for help with acquisition of data.

\section{Funding}

Funding information is not applicable / No funding was received.

\section{Authors' contributions}

MYL provided samples and clinical data. TCH and CYK reviewed tumor sections. YCL designed the experiments, performed the experiments, interpreted the results, and drafted the manuscript. WWC designed the experiments, interpreted the results and made critical revisions to the manuscript. All authors have read and approved the final manuscript.

\section{Ethics approval and consent to participate}

Ethics approval of the study procedures was obtained from the Institutional Review Board of Chung Shan Medical University Hospital via reference number CS2-16003. As no patients were involved and no personal information was used, informed consent was not applicable.

\section{Consent for publication}

Not applicable.

\section{Competing interest}

The authors declare that they have no competing interests.

\section{References}

1. Brown KW, Malik KT. The molecular biology of Wilms tumour. Expert Rev Mol Med. 2001;2001:1-16.

2. Pastore G, Znaor A, Spreafico F, Graf N, Pritchard-Jones K, Steliarova-Foucher E. Malignant renal tumours incidence and survival in European children (1978-1997): report from the Automated Childhood Cancer Information System project. Eur J Cancer. 2006;42(13):2103-14.

3. Steliarova-Foucher E, Colombet M, Ries LAG, Moreno F, Dolya A, Bray F, et al. International incidence of childhood cancer, 2001-10: a population-based registry study. Lancet Oncol. 2017;18(6):719-31.

4. Cabral de Almeida Cardoso L, Rodriguez-Laguna L, Del Carmen Crespo M, Vallespin E, Palomares-Bralo M, MartinArenas R, et al. Array CGH Analysis of Paired Blood and Tumor Samples from Patients with Sporadic Wilms Tumor. PLoS One. 2015;10(8):e0136812.

5. Mahamdallie S, Yost S, Poyastro-Pearson E, Holt E, Zachariou A, Seal S, et al. Identification of new Wilms tumour predisposition genes: an exome sequencing study. Lancet Child Adolesc Health. 2019;3(5):322-31.

6. Treger TD, Chowdhury T, Pritchard-Jones K, Behjati S, et al. The genetic changes of Wilms tumour. Nat Rev Nephrol. 2019;15(4):240-51.

7. Gadd S, Huff V, Walz AL, Ooms A, Armstrong AE, Gerhard DS, et al. A Children's Oncology Group and TARGET initiative exploring the genetic landscape of Wilms tumor. Nat Genet. 2017;49(10):1487-94.

8. Baylin SB. DNA methylation and gene silencing in cancer. Nat Clin Pract Oncol. 2005;2 Suppl 1:S4-11.

9. Lettini AA, Guidoboni M, Fonsatti E, Anzalone L, Cortini E, Maio M. Epigenetic remodelling of DNA in cancer. Histol Histopathol. 2007;22(12):1413-24. 
10. Yamada Y, Yamada Y. The causal relationship between epigenetic abnormality and cancer development: in vivo reprogramming and its future application. Proc Jpn Acad Ser B Phys Biol Sci. 2018;94(6):235-47.

11. Chiang MR, Kuo CW, Wang WC, Hou TC, Kuo CY, Lu MY, et al. Correlations between Histological and Array Comparative Genomic Hybridization Characterizations of Wilms Tumor. Pathol Oncol Res. 2019;25(3):1199-206.

12. Lu MY, Wang WC, Lin CW, Chang A, Lai YC. Identification of a constitutional mutation in the WT1 gene in Taiwanese patients with Wilms tumor. Adv Biosci Biotechnol. 2014;5(3):230-4.

13. Lu MY, Wang WC, Hou TC, Kuo CY, Lai YC. Methylation Statuses of H19DMR and KvDMR at WT2 in Wilms Tumors in Taiwan. Pathol Oncol Res. 2020;26(4):2153-9.

14. Homig-Holzel C, Savola S. Multiplex ligation-dependent probe amplification (MLPA) in tumor diagnostics and prognostics. Diagn Mol Pathol. 2012;21(4):189-206.

15. Wang WC, Lai YC. Genetic analysis results of mature cystic teratomas of the ovary in Taiwan disagree with the previous origin theory of this tumor. Hum Pathol. 2016;52:128-35.

16. Wang WC, Lai YC. Evidence of metachronous development of ovarian teratomas: a case report of bilateral mature cystic teratomas of the ovaries and systematic literature review. J Ovarian Res. 2017;10(1):17.

17. Wagner KJ, Cooper WN, Grundy RG, Caldwell G, Jones C, Wadey RB, et al. Frequent RASSF1A tumour suppressor gene promoter methylation in Wilms' tumour and colorectal cancer. Oncogene. 2002;21(27):7277-82.

18. Harada K, Toyooka S, Maitra A, Maruyama R, Toyooka KO, Timmons CF, et al. Aberrant promoter methylation and silencing of the RASSF1A gene in pediatric tumors and cell lines. Oncogene. 2002;21(27):4345-9.

19. Dubois F, Bergot E, Zalcman G, Levallet G. RASSF1A, puppeteer of cellular homeostasis, fights tumorigenesis, and metastasis-an updated review. Cell Death Dis. 2019;10(12):928.

20. Pfeifer GP, Dammann R. Methylation of the tumor suppressor gene RASSF1A in human tumors. Biochemistry (Mosc). 2005;70(5):576-83.

21. Ehrlich M, Jiang G, Fiala E, Dome JS, Yu MC, Long TI, et al. Hypomethylation and hypermethylation of DNA in Wilms tumors. Oncogene. 2002;21(43): 6694-702.

22. Morris MR, Hesson LB, Wagner KJ, Morgan NV, Astuti D, Lees RD, et al. Multigene methylation analysis of Wilms' tumour and adult renal cell carcinoma. Oncogene. 2003;22(43):6794-801.

23. Ohshima J, Haruta M, Fujiwara Y, Watanabe N, Arai Y, Ariga T, et al. Methylation of the RASSF1A promoter is predictive of poor outcome among patients with Wilms tumor. Pediatr Blood Cancer. 2012;59(3):499-505.

24. Roussel MF. The INK4 family of cell cycle inhibitors in cancer. Oncogene. 1999;18(38):5311-7.

25. Takeuchi S, Matsushita M, Zimmermann M, Ikezoe T, Komatsu N, Seriu T, et al. Clinical significance of aberrant DNA methylation in childhood acute lymphoblastic leukemia. Leuk Res. 2011;35(10):1345-9.

26. Dehennaut V, Loison I, Boulay G, Van Rechem C, Leprince D. Identification of p21 (CIP1/WAF1) as a direct target gene of HIC1 (Hypermethylated In Cancer 1). Biochem Biophys Res Commun. 2013;430(1):49-53.

27. Rathi A, Virmani AK, Harada K, Timmons CF, Miyajima K, Hay RJ, et al. Aberrant methylation of the HIC1 promoter is a frequent event in specific pediatric neoplasms. Clin Cancer Res. 2003;9(10):3674-8.

28. Kruidering M, Evan GI. Caspase-8 in apoptosis: the beginning of "the end"? IUBMB Life. 2000;50(2):85-90.

29. Hsieh P, Yamane K. DNA mismatch repair: molecular mechanism, cancer, and ageing. Mech Ageing Dev. 2008;129(78):391-407.

30. Diniz G, Aktas S, Cubuk C, Ortac R, Vergin C, Olgun N. Tissue expression of MLH1, PMS2, MSH2, and MSH6 proteins and prognostic value of microsatellite instability in Wilms tumor: experience of 45 cases. Pediatr Hematol Oncol. 2013;30(4):273-84.

31. Moller MB, Moller MB, Ino Y, Gerdes AM, Skjodt K, Louis DN, Pedersen NT. Aberrations of the p53 pathway components p53, MDM2 and CDKN2A appear independent in diffuse large B cell lymphoma. Leukemia.

Page 10/11 
1999;13(3):453-9.

32. Arcellana-Panlilio MY, Egeler RM, Ujack E, Pinto A, Demetrick DJ, Robbins SM, et al. Decreased expression of the INK4 family of cyclin-dependent kinase inhibitors in Wilms tumor. Genes Chromosomes Cancer. 2000;29(1):63-9.

33. Roy R, Chun J, Powell SN. BRCA1 and BRCA2: different roles in a common pathway of genome protection. Nat Rev Cancer. 2011;12(1):68-78.

34. Guerra J, Pereira BMS, Cruz Jgvd, Scherer NM, Furtado C, Montalvao de Azevedo R, et al. Genes Controlled by DNA Methylation Are Involved in Wilms Tumor Progression. Cells. 2019;8(8):921.

35. Allenby G, Bocquel MT, Saunders M, Kazmer S, Speck J, Rosenberger M, et al. Retinoic acid receptors and retinoid X receptors: interactions with endogenous retinoic acids. Proc Natl Acad Sci USA. 1993;90(1):30-4.

36. Zhan T, Rindtorff N, Boutros M. Wnt signaling in cancer. Oncogene. 2017;36:1461-73.

37. Schweigert A, Fischer C, Mayr D, von Schweinitz D, Kappler R, Hubertus J. Activation of the Wnt/beta-catenin pathway is common in wilms tumor, but rarely through beta-catenin mutation and APC promoter methylation. Pediatr Surg Int. 2016;32(12):1141-6.

38. Verschuur AC, Vujanic GM, Van Tinteren H, Jones KP, de Kraker J, Sandstedt B. Stromal and epithelial predominant Wilms tumours have an excellent outcome: the SIOP 9301 experience. Pediatr Blood Cancer. 2010;55(2):233-8.

39. Popov SD, Sebire NJ, Vujanic GM. Wilms' Tumour - Histology and Differential Diagnosis. In: van den Heuvel-Eibrink MM (ed) Wilms Tumor, Brisbane (AU): Codon, 2016:3-21.

40. Vujanic GM, Sandstedt B, Harms D, Kelsey A, Leuschner I, de Kraker J, et al. Revised International Society of Paediatric Oncology (SIOP) working classification of renal tumors of childhood. Med Pediatr Oncol. 2002;38(2):79-82.

\section{Figures}

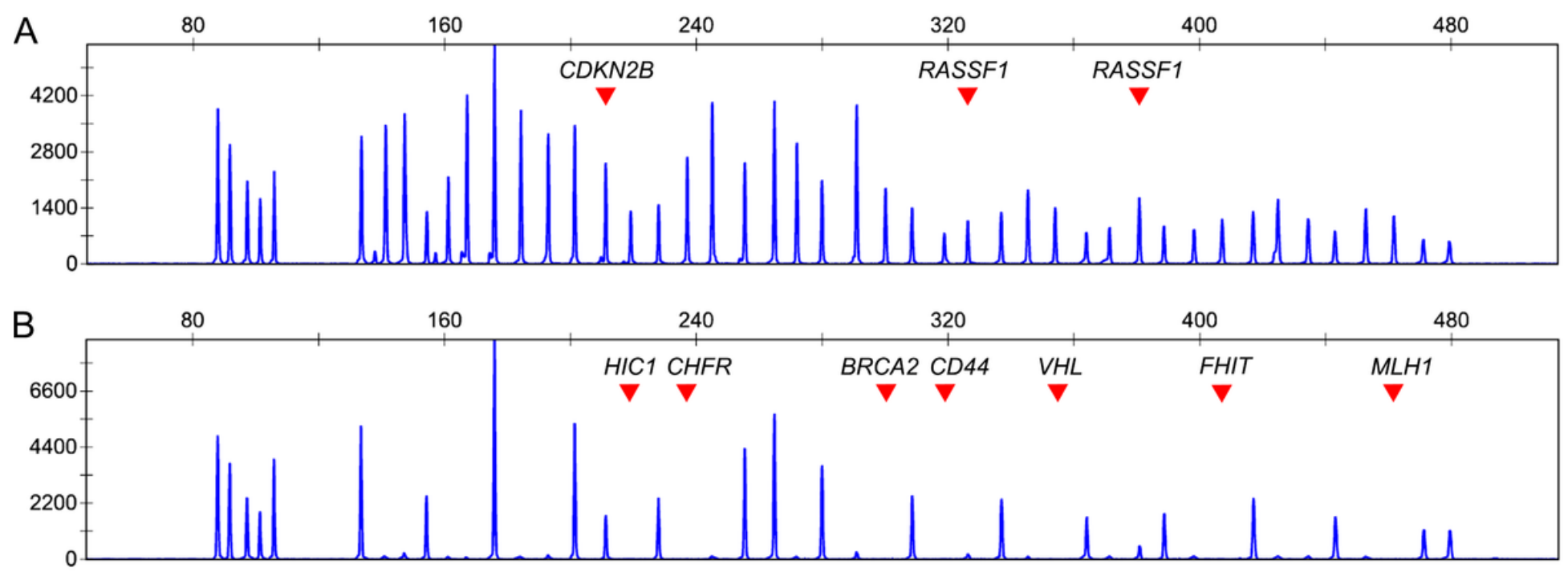

\section{Figure 1}

Detection of the methylation statuses of 24 tumor suppressor genes in W8 by MS-MLPA. (A) The capillary electrophoresis pattern from undigested DNA. Red arrows indicate methylation of RASSF1 and CDKN2B in all six Wilms tumors. (B) The capillary electrophoresis pattern from the same sample but digested with Hhal site. Red arrows indicate that all DNA is unmethylated. Therefore, after MS-MLPA, no signal was generated from the MS-MLPA probes. 